\title{
Contact tracing apps for COVID-19 pandemic: Challenges and potential
}

\author{
Alex Akinbi ${ }^{1}$, Mark Forshaw ${ }^{2}$, Victoria Blinkhorn ${ }^{2}$ \\ o.a.akinbi@ljmu.ac.uk, M.J.Forshaw@ljmu.ac.uk, V.J.Blinkhorn@ljmu.ac.uk
}

\author{
${ }^{1}$ School of Computer Science and Mathematics, James Parsons Building, Liverpool John Moores University, \\ Liverpool, U.K \\ ${ }^{2}$ School of Psychology, Liverpool John Moores University, Tom Reilly Building, Liverpool, U.K
}

\begin{abstract}
The COVID-19 pandemic has spread with increased fatalities around the world and has become an international public health crisis. Public health authorities in many countries have introduced contact tracing apps to track and trace infected persons as part of measures to contain the spread of the Severe Acute Respiratory SyndromeCoronavirus 2 (SARS-CoV-2). However, there are major concerns about its efficacy and privacy with affects mass acceptance amongst a population. This review encompasses the current challenges facing this technology in the fight against the COVID-19 pandemic in neo-liberal societies. We explore and discuss the plausibility for abuse of user privacy rights as such apps collect private user data and can be repurposed by governments for surveillance on their citizens. Other challenges identified and discussed include ethical issues, security vulnerabilities, user behavior and participation, and technical constraints. Finally, in the analysis of this review, recommendations to address these challenges and considerations in the use of less invasive digital contact tracing technologies for future pandemics are presented. For policy makers in neo-liberal societies, this study provides an in-depth review of issues that must be addressed, highlights recommendations to improve the efficacy of such apps, and could facilitate mass acceptance amongst users.
\end{abstract}

Keywords: contact tracing; COVID-19; contact tracing apps; SARS-CoV-2

\section{Introduction}

The novel COVID-19 disease caused by severe acute respiratory syndrome-coronavirus 2 (SARS-CoV-2) has rapidly spread with increased fatalities across the world leading to a worldwide pandemic. In response to the rapidly growing number of cases and the danger of overburdening health systems, many countries have resulted in lockdowns to slow the spread of the novel coronavirus [1]. Other strategies including mass testing and manual contact tracing (using humans or health professionals to collect data) have been deployed to contain the disease and to help ease lockdown restrictions. Like previous similar outbreaks such as Severe Acute Respiratory Syndrome (SARS) and Middle East Respiratory Syndrome (MERS), manual contact tracing and follow-up control measures such as quarantine and isolation were crucially important and successful during these outbreaks [2-5]. Although SARS and MERS were both considered as "fast-course" infectious diseases given their relatively short infectious period, the rate of infection and transmission of the SARS-Cov-2 virus is much faster and death rate outweighs both SARS and MERS which on the scale makes manual contact tracing response labor-intensive, slow and imperfect [6]. One weakness of the manual contact tracing method is that it 
greatly relies on whether the confirmed cases can recollect who they met in the places where they have been or are willing to voluntarily disclose such information. Moreover, there is evidence that SARS-CoV-2 has a reproduction number of around 2-3 in the early stages of an outbreak and many infections can occur without symptoms which are higher than that for SARS (1.7-1.9) and MERS (<1), suggesting that SARS-CoV-2 has a higher pandemic potential which requires a different approach [7-12].

Hence, new technology-based methods have been considered to fill the gap in the identification of contacts, especially if case detection is aggressive [13]. Public health authorities and governments have responded by building digital contact tracing mobile apps like the ones initiated in Singapore, South Korea, and China to keep track of meetings between individuals which allow self-isolation instructions to be sent automatically to everyone when a newly diagnosed patient has interacted with them while infectious with the SARS-CoV-2 virus. Also, Apple and Google in a joint effort to assist announced a new technology for third-party apps on iOS and Android devices to support public health authorities around the world in developing digital contact tracing apps [14]. The concept relies on Bluetooth low-energy (BLE) beaconing technology to record when a phone has come into close proximity with anyone else using the app to track and trace infections. Considering Google Android and Apple iOS jointly hold the highest market share of smartphone operating systems, it seems likely that their approach will be critical in how the majority of contact-tracing apps will function [15]. Although Apple and Google claim user privacy and security are at the core of the design, privacy concerns have also been raised noting that contact tracing apps can otherwise be repurposed to enable unwarranted discrimination and surveillance by governments on their citizens, or data harvesting by third parties [16].

This has created trust issues in the acceptance and participation by users to download and use such apps, hence defeating the real purpose of digital contact tracing. For contact tracing apps to be effective, roughly $50 \%$ to $70 \%$ of a population needs to use them [17] and scientific and epidemiological evidence suggest contact tracing apps have the potential to contribute to reducing the suffering caused by the pandemic and ease lockdown [18]. Strong support for the apps in online surveys carried out in France, Germany, Italy, the UK, and the US, shows that the willingness to install contact tracing apps is very high [19]. However, this does not equate to people doing so. Recent studies show that these apps are not being used by enough of the population in countries including India, Norway, Singapore, and Iceland [20].

In a survey from the Washington Post and the University of Maryland, USA, respondents were split evenly $(50 \%-50 \%)$ on whether they will use a contact tracing app if one was made available. From the 50\% of respondents who said they will use it, only $17 \%$ said they would use it compared to $32 \%$ who said they will probably use it [21]. Despite the threshold set between $50-70 \%$ of the population's use of the app to make it effective, recent statistics from countries where contact tracing apps are voluntary are not promising. At the time of writing, about one million people (20\% of the population) have downloaded Singapore's contact-tracing app 'TraceTogether' with $16 \%$ of the population currently being active users. Australia's 'COVIDSafe' app has been downloaded 6 million times based on data from the Google Play Store (4\% of the population) and so far, just one person has been reported to have been identified using data from it. Similarly, the French contact tracing app 'StopCovid' with 1.9 million downloads from both the App Store and Play Store only managed to send 14 notifications since its release. Recent data from the app developers in the first three weeks since its release show from the 1.9 million downloads, 23,953 people deactivated the app while 460,000 people simply 
uninstalled the app without apparent reasons. The effectiveness of contact tracing apps to track and trace individuals infected with the novel SARS-Cov-2 virus has also been met with skepticism both by industry experts and academics in western democracies.

Considering the above, we conducted an extensive review of the current challenges and application of contact tracing apps in the fight against the COVID-19 pandemic. The aims of this study and the main contributions are as follows:

- Provide an up to date review on the current challenges of contact tracing apps in the fight against COVID-19 in neo-liberal societies;

- Discuss recommendations to address these challenges;

- Explore the current implications of the use of digital contact tracing and in future infectious disease outbreaks.

The remainder of this paper is organized as follows. In Section 2, we describe the methodology used for this study. In Section 3, we discuss the role and potential of contact tracing apps in the fight against COVID-19. The literature survey discussing the challenges of contact tracing apps are discussed in Section 4. In Section 5, we discuss recommendations and implications for its use in future infections disease outbreaks. Finally, in Section 6 we conclude the paper.

\section{Methods}

The extensive literature review was conducted by searching databases of Google Scholar, Web of Science, PubMed, SCOPUS, IEEE Xplore Digital Library, PsychInfo and ScienceDirect using the search terms ("COVID-19" AND "Contact Tracing apps") AND ("COVID-19" AND "Contact Tracing") to identify relevant literature. The searches were run against the title, keywords, or abstract, depending on the search platforms. The searches were conducted between January 1, 2020, through July 30, 2020. Further inputs were also taken from blogs and relevant reports. Previous studies [22-24] have used similar methods to conduct extensive literature surveys and reviews.

The criteria for inclusion included literature written in English, the studies highlight current issues and include commentaries on the existing challenges of contact tracing apps for the COVID-19 pandemic.

\subsection{Review goals}

The purpose of this review is to analyze current and existing studies and their findings and to summarize the current challenges in the application of contact tracing apps in the fight against the COVID-19 pandemic. To make sure our study is focused, we developed three research questions as follows.

1. What are the current challenges facing the efficacy and mass acceptance of contact tracing apps for COVID-19 in neo-liberal societies?

2. What recommendations can be implemented to address these challenges and improve their efficacy mass acceptance?

3. What alternative digital contact tracing methods contrary to the ones available can be adopted now and for future pandemic outbreaks? 


\section{Application of contact tracing apps for the COVID-19 pandemic}

Contact tracing apps can greatly support testing, tracing, isolating, and quarantine measures in the attempt to mitigate and slow the spread of the SARS-Cov-2 virus by speeding up processes of reporting and contact tracing through improved digital data flow, proximity tracing and geolocation tracking [25]. It could play a pivotal role given the ubiquity of internet-connected devices and increase the speed of surveillance of a large population of smartphone users in almost real-time to know where the infection hotspots are. As lockdown measures are gradually being lifted in many countries, contact tracing apps are central to control strategies during the deescalation of social distancing [26]. It can also be crucial in flagging more infections especially in scenarios where manual contact tracing cannot. For example, a yet-to-be diagnosed person has the SARS-Cov-2 virus and takes a crowded bus to work; manual contact tracing is unlikely to identify everyone on the bus standing close to the infected person. Indeed, a study by Kucharski [27] found that combined testing and contact tracing strategies significantly reduced the reproductive number more than mass testing or self-isolation alone.

\subsection{Application of contact tracing apps in East Asia}

In East Asian countries such as China and especially Taiwan, contact tracing apps have been mandatory and proved effective alongside manual contact tracing methods in identifying new cases since the end of lockdown $[28,29]$. The apps generally work by assigning a color code (green, yellow, or red) using an algorithmic assessment of the user's travel history and health status. People who can show a green health code on their smartphones demonstrate they have not been in contact with a confirmed case of COVID-19 [30-32]. In South Korea, contact tracing apps such as the 'Corona 100' seems to be popular and has enabled public health officials to reduce the time needed to trace a patient's movements from around 24 hours to approximately 10 minutes and thus, helped the general public avoid infectious areas.

South Korea's extensive contact tracing, testing, and isolation measures received overwhelming support by the population and have helped to reduce the virus's spread [33]. Laws passed and data transparency during the MERS outbreak in 2015 allows the South Korean government to collect and publish public data including travel histories of confirmed patients. Hence, privacy of the population has been given up to the government especially in response to public health safety. To ensure people under compulsory home quarantines do not stray from the confines of their apartments, the Hong Kong government required compulsory download of the 'StayHomeSafe' app and provided geofencing electronic tracker wristbands that alert authorities if they violated quarantines. These approaches have significantly contributed to the mass acceptance of contact tracing apps so far in the region.

There is little evidence to suggest that the use of these types of approaches adopted in East Asian countries might be successfully transferable to neo-liberal societies with different political and cultural systems. The barrier appears to be that many of these countries are very sensitive to privacy issues and privacy is protected by law like the General Data Protection Regulation (GDPR) [34,35]. A study by Hernandez-Orallo [36] shows that for possible second waves of infection, contact tracing apps can be effective in controlling the SARS-Cov-2 virus, assuming that a percentage of the population will have gained immunity, or implemented in combination with some other lenient measures, such as social distancing. Moreover, for many of such countries that are resuming business operations and social activities, or where protests are happening and the number of social contacts increases, it will be worthwhile for them to invest in strategies to vastly improve the mass acceptance of 
contact-tracing apps to enable rapid response to a resurgence of the SARS-Cov-2 virus [37]. However, the apparent dilemma faced is making a conscious choice between privacy and public health whilst showing the efficacy of such apps.

\section{Challenges of contact tracing apps in neo-liberal societies}

The decision to develop and deploy contact tracing apps for tracking and tracing the COVID-19 pandemic continues to raise data privacy concerns and a balance between user data privacy and societal benefit has been considered [38]. This coupled with its effectiveness, ethical considerations, security risks, and technical issues has been highlighted as major challenges affecting mass acceptance amongst a population in neo-liberal societies. In this section, we present an extensive literature survey of the major challenges affecting contact tracing apps in the fight against COVID-19.

\subsection{User data privacy concerns}

Privacy concerns related to user data has been an issue affecting the acceptance and participation in the use of contact tracing apps. Questions such as how user data will be anonymized, where the data will be stored, who has access to the data, how it will be shared, used and destroyed when the pandemic is over have been subjects of huge debate. Parker [18] highlights that justification for privacy infringements of users is hypothetically justifiable in cases where contact tracing apps have the potential to contribute to the saving of many lives and reduce enormous suffering caused by a blanket population lockdown. Hence, people should be prepared to trade-off privacy encroaching contact tracing apps for civil liberty and see it as a public duty to save lives as lockdown is being eased.

An app encroaching on people's privacy while providing little contribution compared to other measures in tackling the spread of COVID-19 would be ethically dubious [39]. Mandatory and coercive installation of contact tracing apps by residents and foreigners despite privacy issues and civil liberty encroachment with the possibility of violators facing severe jail time or fines have been seen in several countries including China, Qatar, South Korea, Poland, Russia, Kazakhstan, and India [40]. This has made a significant number of the population use the apps compared to western democracies where there is no legal obligation. In South Korea, local authorities have gone as far as using surveillance techniques which includes the use of Global Positioning System (GPS), credit card transaction records, closed-circuit television (CCTV) footage and medical records to monitor movements of contacts when a patient with COVID-19 is detected [41].

The lack of trust in government and their motive appears to be a key factor that creates a negative effect on people's decisions to install a contact tracing app on their phones especially in neo-liberal societies where the use is not mandatory and success depends on the establishment of sustained and sound public trust and confidence [19]. Lack of the public's trust in governments has been impacted since Edward Snowden's revelations on government global surveillance, especially in the USA in the post 9-11 era [42]. Other approaches suggested in Kapa et al. [38], highlight a privacy-first scenario (centralized model) where the central authority (public health authorities) collecting data from such apps never know who the data originated from, but can identify if the data was from an infected individual or not. 
In such a scenario, the data would need to be encrypted but users are still required to share identifiable personal information. An example is the 'TraceTogether' app, developed for the government of Singapore, which requires all users to share their contact information with the app's administrators while in Argentina, the app for self-diagnosis requires people to include their National ID, email and phone number. This way authorities can identify contacts and keep track of infected locations with very little private information shared. Another modeled scenario (decentralized model) would be where no central authority is aware of the identities of infected individuals, but users can still be made aware of potential exposure through a database that doesn't store as much information about the app's users and they can respond accordingly. This is the approach promoted by Google and Apple but has limitations as public health authorities do not get anonymous aggregated data to identify hotspots or predict future outbreaks.

Apart from privacy issues associated with central authorities' access to user data, concerns associated with access to user data by third parties have also been raised. These include any individual with whom a user has exchanged tokens in the contact tracing app based on some notion of physical proximity, big data analysis companies, or malicious actors where the contact tracing app's system is naïve or vulnerable to information leakage [43]. In May 2020, the official COVID-19 contact tracing app for the state of North Dakota, USA, was found to send user location data and the unique user identifier to Foursquare and other data to Google including a bug-tracking company with the users' consent [44]. Technical analysis of 11 contact tracing apps revealed the use of mass surveillance tools in Bahrain's 'BeAware Bahrain', Kuwait's 'Shlonik' and Norway's 'Smittestopp' apps with all three actively carrying out live or near-live tracking of users' locations by frequently uploading GPS coordinates to a central server [45]. A scientific data protection impact assessment (DPIA) of contact tracing app designs (including centralized and decentralized models) conducted by Bock [46] found that none of the proposed designs ensures proper anonymization, and that informed consent would not be a legitimate legal ground for the processing, that data subjects' rights are not sufficiently safeguarded and that no design provides for sufficient purpose-binding. The issues highlighted show that maintaining the balance between user privacy and public health is a huge challenge for contact tracing apps in the fight against COVID19.

\subsection{Ethical considerations}

In many countries, residents have been living under lockdown with their civil liberties heavily curtailed. Many businesses have been forced to stop operations forcing millions out of work. According to the International Monetary Fund's (IMF) April World Economic Outlook, the global growth in 2020 is expected to fall to -3\% making it the worst recession since the Great Depression [47]. The United Kingdom's economy GDP is projected to fall by $11.5 \%$ while the USA, China, Germany, and France GDPs are predicted to fall by $6.6 \%$, $2.6 \%, 6.6 \%$, and $11.4 \%$ respectively [48]. As countries slowly lift restrictions and business open to enable quick economic recovery, Klenk et al. [49] suggest that digital contact tracing itself can contribute to general fairness risk associated with discriminate mitigation measures. For example, disadvantaged workers are less likely to be able to work from home, engaging in work means that they are at higher risks of becoming infected and are more likely to form social ties with others in similarly precarious arrangements. Therefore, they may be forced to quarantine simply because they have been in close proximity with others in the same social group although they may not be at high risk of infection. The lack of smartphones and internet access, as well as share 
of informal employment all come together to disproportionately impact the low income communities which continue to drive the health divide rooted in social status and economic differences even further [40].

Bock and colleagues [46] discuss the risk of data collected through contact tracing apps by public health authorities and governments can be used not just for epidemiological studies but also for behavioral profiling of a population. They explain that the behavioral profiles if correlated with other demographic and socio-economic data may motivate selective policies in which a population that has been measured as being on average more willing to take risks are treated differently by future restrictions than other groups whose compliance is supposedly higher. This raises serious ethical issues as data can be used to discriminate against a population or geographic locations where cases of COVID-19 are higher. For example, behavioral scoring can be used to determine access to medical resources, funding, treatment, etc. It was discovered that Bahrain's 'BeAware Bahrain' app has been sharing data with a national television show called Are You at Home? which offered prizes to those who stayed at home during Ramadan [50]. In South Korea, contact-tracing laws permit the government to determine the immigration status of infected individuals. Sinha and Paterson [15] highlight that if such laws exist in the U.S., undocumented communities may not seek healthcare and that over time the same technologies and laws could be used to track undocumented migrants.

\subsection{Security vulnerabilities}

Security flaws in the design and implementation of contact tracing apps have the potential to put sensitive personal details of users at risk. A recent investigation by Amnesty Security Lab discovered a significant weakness in the configuration of Qatar's mandatory EHTERAZ contact tracing app (Amnesty International UK, 2020). The vulnerability could allow hackers access to highly sensitive personal information, including the name, national ID, health status, and location data of more than one million users. Similar vulnerabilities have been disclosed in India's 'Aarogya Setu' and Pakistan's 'Covid-19 Gov PK' apps. These vulnerabilities were from apps that have been tested by security researchers so far. Other proposed contact tracing apps may have similar or different security flaws that make them susceptible to attacks and data leaks.

Dar [52] described possible attacks such as Bluejacking, Bluesnarfing, and Bluebugging attacks on contact tracing apps that use Bluetooth technology. Recent Bluetooth vulnerabilities which include BlueFrag (CVE2020-0022 which affected Android devices running Android 8.0 to 9.0) and Bluetooth BIAS Attack (affected multiple Android and iOS devices) [53] were disclosed in February and May 2020 respectively and required patching. However, many Android devices did not receive this update as more than one billion Android devices around the world are no longer supported by security updates, leaving them potentially vulnerable to attacks. According to Google's data from 2019, around 40\% of Android active users worldwide are on version 6.0 or earlier and no longer receive security updates [54]. A successful Bluetooth BIAS attack would allow a malicious actor to impersonate a device from a previous secure Bluetooth connection pairing between two devices (see Figure 1). This can be leveraged to conduct social engineering attacks or take control of the vulnerable device as contact tracing apps always require Bluetooth enabled to function. 


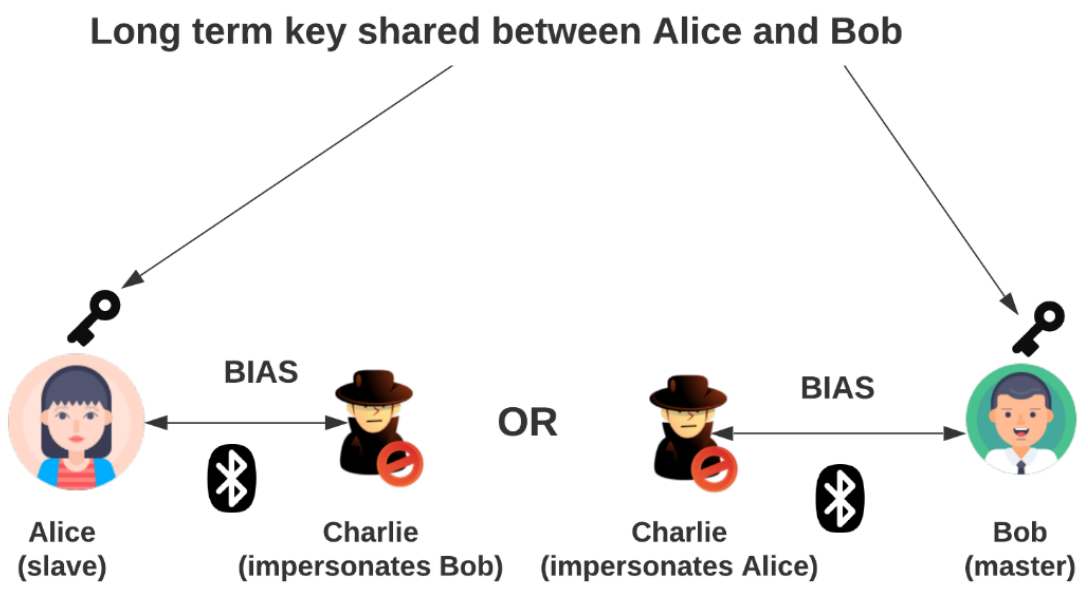

Figure 1. Bluetooth Impersonation Attacks (BIAS)

\subsection{Technical constraints}

The development and roll-out of contact tracing apps revolve around several assumptions that raise questions about its efficacy to advance public health in the fight against COVID-19. These assumptions are that a large percentage of the population have access to compatible smartphones and an internet connection, that Bluetooth signals are accurate, and that people will choose to install and use the apps [40]. According to Statista, the current number of smartphone users in the world today is 3.5 billion, and this means $44.98 \%$ of the world's population owns a smartphone [55]. From this percentage, smartphones running the Android operating system held an $87 \%$ share of the global market in 2019 compared to the mobile operating system developed by Apple (iOS), which had a 13\% share of the market [56], thus making Android the most popular mobile operating system used across the world. However, more than one billion Android devices are two years or more out of date and do not receive updates from device manufacturers and carriers [57]. This means many Android devices may not benefit from updates to the new COVID-19 contact tracing system Google is building in collaboration with Apple.

Access to mobile internet across the world is not evenly distributed. According to the latest report from the GSMA Mobile Economy[58], smartphone subscriber penetration is considerably low in Sub-Saharan Africa (45\%) compared to other regions like Europe (86\%), North America (83\%), Greater China (82\%) and Asia Pacific (60\%). This disparity is closely related to GDP per capita of countries where citizens from poorer countries are less likely to own a smartphone. Data from the World Bank from 2017[59], shows the mobile phone subscription vs GDP per capita from countries in Africa, especially Sub-Saharan Africa falls well behind compared to countries from other regions (see Figure 2). Hence, many countries in the region would not benefit from the roll-out of contact tracing apps, risk getting infected, or could be subject to extensive periods of lockdown. 
Even in regions like Europe, not all households have access to mobile internet and smartphones. In the UK, figures released by the Office for National Statistics (ONS), show that a third of households still do not have access to mobile broadband [60]. The latest figures in 2020 show that $30 \%$ of UK senior citizens aged 55 years and above do not own or have access to a smartphone and an estimated $21 \%$ of young adults aged 18 years and above do not have a smartphone [61].

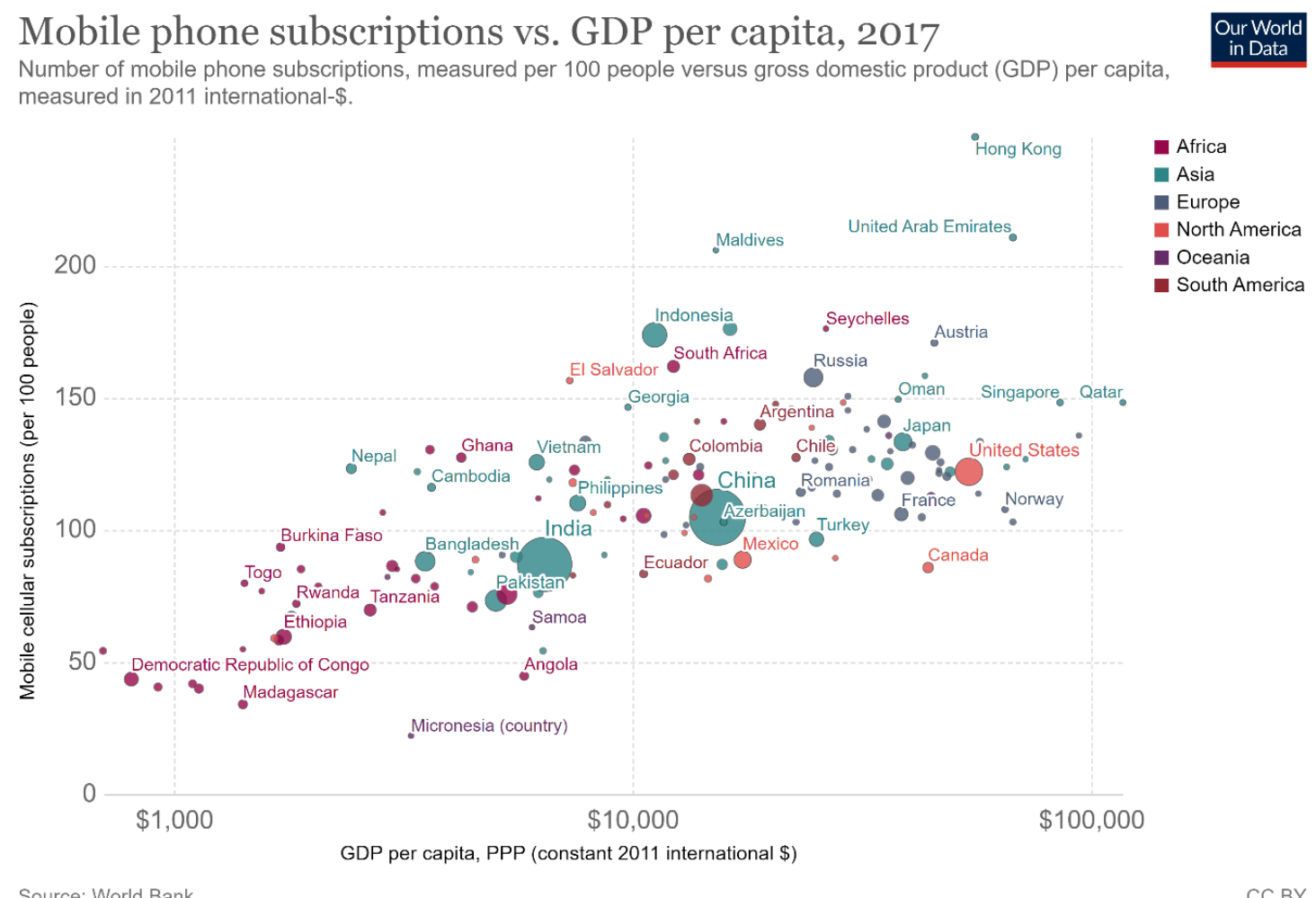

Figure 2. Mobile phone subscriptions vs. GDP per capita, 2017 ( image source: Our World In Data [59])

Bluetooth signals suffer from accuracy problems and will require developers to fine-tune how signals will be transmitted by lowering the transmission power to prevent such long-distance reception. Magklaras [62] highlighted different Bluetooth versions and smartphone chipset implementations that can result in different operational and information security aspects of its use for contact tracing. Signal strength can vary significantly depending on the relative orientation of smartphones, on absorption by the human body, reflection, or absorption of radio signals in buildings and on trains. Table 1 shows the differences between the versions of Bluetooth.

According to the inventors of Bluetooth, Jaap Haartsen, and Sven Mattisson, the signal's path loss will vary significantly depending on extenuating conditions (free space or obscured) [63]. Duration, proximity, and direction of signal strength between two devices would also need to be measured to deal with problems associated with false positives. Especially in scenarios where the contact tracing app detects a non-valid exposure or false negative, where the app fails to detect a valid exposure because the distance was miscalculated, or even because of other external factors and extenuating circumstances. The inevitable danger of 
non-valid exposure measurements is linked to the risk that users are wrongly isolated, potentially several times in succession, with considerable economic and social consequences to those affected [46]. The SARS-CoV-2 virus could spread by touching an object or surface with a virus present from an infected person, and then touching the mouth, nose, or eyes. Hence in such cases, a contact tracing app will not be able to detect this and may give people a false sense of security.

Table 1. Comparison of Bluetooth versions

\begin{tabular}{|l|l|l|l|}
\hline & Bluetooth v2.1 & Bluetooth v4.0 (LE) & Bluetooth v5.0 (LE) \\
\hline Range & Up to $100 \mathrm{~m}$ & Up to $100 \mathrm{~m}$ & Up to $400 \mathrm{~m}$ \\
\hline Max Range & $\sim 100 \mathrm{~m}$ (outdoors) & $\sim 100 \mathrm{~m}$ (outdoors) & $\sim 1000 \mathrm{~m}$ (outdoors) \\
\hline Frequency & $2.402-2.481 \mathrm{GHz}$ & $2.402-2.481 \mathrm{GHz}$ & $2.402-2.481 \mathrm{GHz}$ \\
\hline Max data rate & $1-3 \mathrm{Mbit} / \mathrm{s}$ & $1 \mathrm{Mbit} / \mathrm{s}$ & $2 \mathrm{Mbit} / \mathrm{s}$ \\
\hline Application Throughput & $0.7-2.1 \mathrm{Mbit} / \mathrm{s}$ & Up to $305 \mathrm{kbit} / \mathrm{s}$ & Up to $1,360 \mathrm{kbit} / \mathrm{s}$ \\
\hline Topologies & Point to Point, scatternet & Point to point, mesh network & Point to point, mesh network \\
\hline Network Standard & IEEE 802.15 .1 & IEEE 802.15 .1 & IEEE 802.15 .1 \\
\hline
\end{tabular}

\subsection{Problems associated with user behavior and participation}

Most citizens over the age of 65 are not tech-savvy compared to the younger generation. Using the latest data on smartphone usage by age, sourced from the UK's communications regulator OFCOM, there is a digital divide between adults of certain age groups and their attitudes towards the use of smartphones with young adults more likely to use smartphones compared to senior citizens in the UK. Using the latest data on smartphone usage by age, sourced from OFCOM, 3\% of adults in the UK between ages 65-74 years only use a smartphone to go online compared to $16 \%$ and $20 \%$ of adults between the ages 16- 24 and 25- 34 years respectively [64]. While almost all adults aged 16 to 44 years used the Internet daily, or almost every day (99\%), the older age groups, 65 years and above used it less frequently. Of this older age group, $61 \%$ of adults aged 65 years and above, used the Internet daily, while $24 \%$ had not used the Internet in the last three months. Access to technologies remains a key issue. If the behavior is to be driven in the direction of uptake, then the technologies need to be ubiquitous. Furthermore, the same OFCOM report shows that, amongst smartphone users, data consumption and battery usage are key issues, which could be problematic if the public is being asked to adopt a technology that could add to battery depletion and require additional data to be used if location is switched on. If the public feels that the use of an app would have marginal costs associated with it, then this could lead to a decrease in uptake.

The success of any voluntarily installed app, for whatever purpose, is dependent upon user buy-in. There are several dimensions to that judgment of acceptability of technology, including its benefits and disbenefits, the moral or social imperatives, the perceived efficacy of the app, and the behavior of significant others in the lives of the potential user. Psychological research into the adoption of technologies focuses on these issues; where wetware meets hardware and software. These are challenging situations based on social dilemmas that involve a conflict between immediate self-interest and longer-term collective interests [65]. Based on reviewing 17 social dilemma studies involving more than 7,000 individuals conducted by Thoni and Volk [66], the majority (about $60 \%$ ) are known as "conditional cooperators", are very sensitive, tend to cooperate if they believe others will cooperate and will reduce cooperation as soon as they realize a few others are not complying with agreed rules. 
Hence based on social dilemmas, many individuals may choose to use contact tracing apps if they believe others will. Therefore, public health authorities must pass the right message across to ensure the beliefs of conditional co-operators are reassured in maintaining their commitment to using such apps.

A study by Trang et al. [67] concluded that it is difficult for policy makers to design one app that fits all individuals in a society. Especially when the propensity to accept such non-mandatory apps varies between critics, advocates, and undecided individuals amongst the neo-liberal population. Straub [68] reviewed other research testing theories of adoption of behaviors and technologies, focusing on certain specific theories, but with no reference to any of the kinds of technologies relevant to virus tracking. Calvo et al. [69] wrote an editorial to the effect that tracking or screening technologies can work when the public understands the value of them for their health and wellbeing, and both parties are signed up to that greater purpose. Under such conditions, surveillance concerns are minimized. This is confirmed by Williams et al [70]in a qualitative, focusgroup study.

Bradshaw et al. [71] report a study of intentions to use a contact-tracing app in Australia, with the conclusion that uptake of the software can be increased if the security concerns are addressed, but that message framing did not make a significant difference to intention when autonomy-controlling and supportive messages were compared. Supportive messages involve choice and freedom, whereas autonomy-controlling messages use model verbs such as "should" and "must". However, the study does suffer from the intention-behavior gap reported by psychologists for decades [72].

\section{Recommendations and future considerations}

Maintaining a balance especially between user privacy and societal benefit is a huge challenge if digital contact tracing using mobile apps is to succeed in the fight against COVID-19. Also, influencing user behavior in the participation, and dealing with technical constraints associated with the underlying technology is essential if contact tracing apps are to succeed now and in respect of dealing with future pandemics. In this section, we discuss recommendations in addressing some of these issues and future considerations in the development and implementation of digital contact tracing.

Data collected from such apps should only be used to support public health measures, the source code should be made public and subjected to public analysis and finally, its use must be voluntary, used with the explicit consent of the user and the systems must be designed to be able to be switched off, and all data deleted when the current pandemic is over [73]. There are suggestions that a non-partisan independent committee with representatives from legal, health, and privacy experts should be established to oversee the development of contact tracing apps, its information ecosystem, and data governance. Only anonymized aggregated data should be shared with public health authorities and any personal identifiable information must be deleted once the pandemic is over [74].

On issues associated with Bluetooth, it is recommended that the Bluetooth LE signal should be regulated in a standardized manner when operating a contact tracing app so that the effective range of the protocol is reduced [62]. Legislation should be put in place which mandates smartphone manufacturers and carriers to provide critical system updates especially for Android devices vulnerable to critical Bluetooth vulnerabilities such as BlueFrag and BIAS attack. 
In conclusion, we know very little for sure about the psychological factors that would influence the adoption of an app for contact tracing at present for the ongoing outbreak of COVID-19, but there are findings from relevant research more widely which suggest that people would respond best to messages which alleviate their concerns over security, emphasize personal autonomy, and where the societal benefits are clearly articulated. It is also important that policy makers study their demographic to understand user perception. Designing a contact tracing app that targets most of the population and addresses their concerns ( privacy and usability) can increase mass acceptance. Moreover, to realize their intended societal benefits, contact tracing apps require mass acceptance [67].

For future considerations, the use of less invasive technologies such as Artificial Intelligence (AI) has been suggested and can help analyze the level of infection by the SARS-CoV-2 virus by identifying hotspots, tracing, and monitoring infected persons [24,75,76]. Other methods include the use of thermal-based imaging using the Internet of Medical Things (IoMT) to trace and track positive cases and help control the spread of COVID-19 infection and future infectious disease outbreaks [77,78].

\section{Conclusion}

The impact of the COVID-19 pandemic represents an unprecedented challenge to public health authorities and respective governments across the world. This has brought severe pressure on health services and introduced radical changes to the way of life for both individuals and organizations. In a way to stop the infection of the SARS-CoV-2 virus spreading, public health authorities have considered and introduced robust contact tracing systems which include the use of digital contact tracing apps. In this paper, we discussed the application of contact tracing apps in East Asia and the challenges faced by neo-liberal societies in their use to fight against the COVID-19 pandemic. Although contact tracing apps are a promising technology for rapid tracing and tracking of infected persons, it can support manual contact tracing and tracking methods in the control of the SARSCoV-2 virus. However, many people have an intrinsic mistrust of the government especially in neo-liberal societies and are concerned that the use of contact tracing apps could be the beginning of more pervasive government surveillance. Also, since these apps are not mandatory, it is difficult to predict mass acceptance and participation. If contact tracing apps are to succeed, it is important governments and policy makers gain the trust of their citizens and show adequate transparency in how user data is collected and used. Its efficacy and how these challenges are currently addressed in the fight against this novel disease will determine the role of digital contact tracing technologies in future pandemic outbreaks and what lessons can be learned from identified inadequacies.

\section{Acknowledgments}

This research did not receive any specific grant from funding agencies in the public, commercial, or not-forprofit sectors.

\section{References}

[1] PEPP-PT, Pan-European Privacy-Preserving Proximity Tracing, PEPP-PT. (2020). https://www.pepppt.org/content (accessed April 24, 2020).

[2] K.O. Kwok, A. Tang, V.W.I. Wei, W.H. Park, E.K. Yeoh, S. Riley, Epidemic Models of Contact 
Tracing: Systematic Review of Transmission Studies of Severe Acute Respiratory Syndrome and Middle East Respiratory Syndrome, Comput. Struct. Biotechnol. J. (2019).

doi:10.1016/j.csbj.2019.01.003.

[3] S. Riley, C. Fraser, C.A. Donnelly, A.C. Ghani, L.J. Abu-Raddad, A.J. Hedley, G.M. Leung, L.M. Ho, T.H. Lam, T.Q. Thach, P. Chau, K.P. Chan, S.V. Lo, P.Y. Leung, T. Tsang, W. Ho, K.H. Lee, E.M.C. Lau, N.M. Ferguson, R.M. Anderson, Transmission dynamics of the etiological agent of SARS in Hong Kong: Impact of public health interventions, Science (80-. ). (2003). doi:10.1126/science.1086478.

[4] S. Saurabh, S. Prateek, Role of contact tracing in containing the 2014 Ebola outbreak: A review, Afr. Health Sci. (2017). doi:10.4314/ahs.v17i1.28.

[5] K.-I. Leong, Y.-W. Si, R.P. Biuk-Aghai, S. Fong, Contact tracing in healthcare digital ecosystems for infectious disease control and quarantine management, in: 2009 3rd IEEE Int. Conf. Digit. Ecosyst. Technol., IEEE, 2009: pp. 306-311. doi:10.1109/DEST.2009.5276730.

[6] K. Sun, C. Viboud, Impact of contact tracing on SARS-CoV-2 transmission, Lancet Infect. Dis. (2020). doi:10.1016/S1473-3099(20)30357-1.

[7] A.J. Kucharski, T.W. Russell, C. Diamond, Y. Liu, J. Edmunds, S. Funk, R.M. Eggo, F. Sun, M. Jit, J.D. Munday, N. Davies, A. Gimma, K. van Zandvoort, H. Gibbs, J. Hellewell, C.I. Jarvis, S. Clifford, B.J. Quilty, N.I. Bosse, S. Abbott, P. Klepac, S. Flasche, Early dynamics of transmission and control of COVID-19: a mathematical modelling study, Lancet Infect. Dis. (2020). doi:10.1016/S14733099(20)30144-4.

[8] X. He, E.H.Y. Lau, P. Wu, X. Deng, J. Wang, X. Hao, Y.C. Lau, J.Y. Wong, Y. Guan, X. Tan, X. Mo, Y. Chen, B. Liao, W. Chen, F. Hu, Q. Zhang, M. Zhong, Y. Wu, L. Zhao, F. Zhang, B.J. Cowling, F. Li, G.M. Leung, Temporal dynamics in viral shedding and transmissibility of COVID-19, Nat. Med. (2020). doi:10.1038/s41591-020-0869-5.

[9] N. Petrosillo, G. Viceconte, O. Ergonul, G. Ippolito, E. Petersen, COVID-19, SARS and MERS: are they closely related?, Clin. Microbiol. Infect. (2020). doi:10.1016/j.cmi.2020.03.026.

[10] J.T. Wu, K. Leung, G.M. Leung, Nowcasting and forecasting the potential domestic and international spread of the 2019-nCoV outbreak originating in Wuhan, China: a modelling study, Lancet. (2020). doi:10.1016/S0140-6736(20)30260-9.

[11] T. Liu, J. Hu, M. Kang, L. Lin, H. Zhong, J. Xiao, G. He, T. Song, Q. Huang, Z. Rong, A. Deng, W. Zeng, X. Tan, S. Zeng, Z. Zhu, J. Li, D. Wan, J. Lu, H. Deng, J. He, W. Ma, Transmission Dynamics of 2019 Novel Coronavirus (2019-nCoV), SSRN Electron. J. (2020). doi:10.2139/ssrn.3526307.

[12] H. Chen, B. Yang, H. Pei, J. Liu, Next Generation Technology for Epidemic Prevention and Control: Data-Driven Contact Tracking, IEEE Access. 7 (2019) 2633-2642. doi:10.1109/ACCESS.2018.2882915.

[13] L. Ferretti, C. Wymant, M. Kendall, L. Zhao, A. Nurtay, D.G. Bonsall, C. Fraser, Quantifying dynamics of SARS-CoV-2 transmission suggests that epidemic control and avoidance is feasible through instantaneous digital contact tracing, MedRxiv. (2020). doi:10.1101/2020.03.08.20032946.

[14] A. Vaughan, The problems with contact-tracing apps, New Sci. (2020). doi:10.1016/s02624079(20)30787-9.

[15] P. Sinha, A.E. Paterson, Contact tracing: Can 'Big tech' come to the rescue, and if so, at what cost?, EClinicalMedicine. (2020) 100412. doi:10.1016/j.eclinm.2020.100412.

[16] A. Hearn, Digital contact tracing will fail unless privacy is respected, experts warn, Guard. Newsp. (2020). https://www.theguardian.com/world/2020/apr/20/coronavirus-digital-contact-tracing-will-failunless-privacy-is-respected-experts-warn (accessed June 11, 2020).

[17] S. Fussel, W. Knight, The Apple-Google Contact Tracing Plan Won't Stop Covid Alone, Wired.Com. (2020). 24/04/2020.

[18] M.J. Parker, C. Fraser, L. Abeler-Dörner, D. Bonsall, Ethics of instantaneous contact tracing using mobile phone apps in the control of the COVID-19 pandemic, J. Med. Ethics. 46 (2020) 427-431. doi:10.1136/medethics-2020-106314. 
[19] S. Altmann, L. Milsom, H. Zillessen, R. Blasone, F. Gerdon, R. Bach, F. Kreuter, D. Nosenzo, S. Toussaert, J. Abeler, Acceptability of app-based contact tracing for COVID-19: Cross-country survey evidence, MedRxiv. (2020). doi:10.1101/2020.05.05.20091587.

[20] S. Findlay, S. Palma, Coronavirus contact-tracing apps struggle to make an impact, Financ. Times. (2020). https://www.ft.com/content/21e438a6-32f2-43b9-b843-61b819a427aa (accessed June 11, 2020).

[21] W. Roper, Americans Split on Contact Tracing App, Statista. (2020).

https://www.statista.com/chart/21573/contact-tracing-app-adoption/ (accessed June 16, 2020).

[22] S. Swayamsiddha, C. Mohanty, Application of cognitive Internet of Medical Things for COVID-19 pandemic, Diabetes Metab. Syndr. Clin. Res. Rev. 14 (2020) 911-915. doi:10.1016/j.dsx.2020.06.014.

[23] R.P. Singh, M. Javaid, A. Haleem, R. Suman, Internet of things (IoT) applications to fight against COVID-19 pandemic, Diabetes Metab. Syndr. Clin. Res. Rev. 14 (2020) 521-524. doi:10.1016/j.dsx.2020.04.041.

[24] R. Vaishya, M. Javaid, I.H. Khan, A. Haleem, Artificial Intelligence (AI) applications for COVID-19 pandemic, Diabetes Metab. Syndr. Clin. Res. Rev. (2020). doi:10.1016/j.dsx.2020.04.012.

[25] K.N. Vokinger, V. Nittas, C.M. Witt, S.I. Fabrikant, V. von Wyl, Digital health and the COVID-19 epidemic: an assessment framework for apps from an epidemiological and legal perspective, Swiss Med. Wkly. (2020). doi:10.4414/smw.2020.20282.

[26] M.E. Kretzschmar, G. Rozhnova, M. Bootsma, M.E. van Boven, J. van de Wijgert, M. Bonten, Time is of the essence: impact of delays on effectiveness of contact tracing for COVID-19, MedRxiv. (2020). doi:10.1101/2020.05.09.20096289.

[27] A.J. Kucharski, P. Klepac, A.J.K. Conlan, S.M. Kissler, M.L. Tang, H. Fry, J.R. Gog, W.J. Edmunds, J.C. Emery, G. Medley, J.D. Munday, T.W. Russell, Q.J. Leclerc, C. Diamond, S.R. Procter, A. Gimma, F.Y. Sun, H.P. Gibbs, A. Rosello, K. van Zandvoort, S. Hué, S.R. Meakin, A.K. Deol, G. Knight, T. Jombart, A.M. Foss, N.I. Bosse, K.E. Atkins, B.J. Quilty, R. Lowe, K. Prem, S. Flasche, C.A.B. Pearson, R.M.G.J. Houben, E.S. Nightingale, A. Endo, D.C. Tully, Y. Liu, J. Villabona-Arenas, K. O’Reilly, S. Funk, R.M. Eggo, M. Jit, E.M. Rees, J. Hellewell, S. Clifford, C.I. Jarvis, S. Abbott, M. Auzenbergs, N.G. Davies, D. Simons, Effectiveness of isolation, testing, contact tracing, and physical distancing on reducing transmission of SARS-CoV-2 in different settings: a mathematical modelling study, Lancet Infect. Dis. (2020). doi:10.1016/S1473-3099(20)30457-6.

[28] H.-Y. Cheng, S.-W. Jian, D.-P. Liu, T.-C. Ng, W.-T. Huang, H.-H. Lin, Contact Tracing Assessment of COVID-19 Transmission Dynamics in Taiwan and Risk at Different Exposure Periods Before and After Symptom Onset, JAMA Intern. Med. (2020). doi:10.1001/jamainternmed.2020.2020.

[29] C.J. Wang, C.Y. Ng, R.H. Brook, Response to COVID-19 in Taiwan, JAMA. 323 (2020) 1341. doi:10.1001/jama.2020.3151.

[30] J. Abeler, M. Bäcker, U. Buermeyer, H. Zillessen, Covid-19 contact tracing and data protection can go together, JMIR MHealth UHealth. (2020). doi:10.2196/19359.

[31] C.J. Wang, C.Y. Ng, R.H. Brook, Response to COVID-19 in Taiwan: Big Data Analytics, New Technology, and Proactive Testing, JAMA - J. Am. Med. Assoc. (2020). doi:10.1001/jama.2020.3151.

[32] R. Steinbrook, Contact Tracing, Testing, and Control of COVID-19-Learning From Taiwan, JAMA Intern. Med. (2020). doi:10.1001/jamainternmed.2020.2072.

[33] M. Zastrow, South Korea is reporting intimate details of COVID-19 cases: has it helped?, Nature. (2020). https://www.nature.com/articles/d41586-020-00740-y (accessed August 12, 2020).

[34] C.L. Miltgen, D. Peyrat-Guillard, Cultural and generational influences on privacy concerns: a qualitative study in seven European countries, Eur. J. Inf. Syst. 23 (2014) 103-125. doi:10.1057/ejis.2013.17.

[35] F. Rowe, Contact tracing apps and values dilemmas: A privacy paradox in a neo-liberal world, Int. J. Inf. Manage. (2020) 102178. doi:10.1016/j.ijinfomgt.2020.102178.

[36] E. Hernandez-Orallo, P. Manzoni, C.T. Calafate, J.-C. Cano, Evaluating How Smartphone Contact Tracing Technology Can Reduce the Spread of Infectious Diseases: The Case of COVID-19, IEEE 
Access. 8 (2020) 99083-99097. doi:10.1109/ACCESS.2020.2998042.

[37] C.R. MacIntyre, Case isolation, contact tracing, and physical distancing are pillars of COVID-19 pandemic control, not optional choices, Lancet Infect. Dis. (2020). doi:10.1016/S1473-3099(20)305120 .

[38] S. Kapa, J. Halamka, R. Raskar, Contact Tracing to Manage COVID19 Spread - Balancing Personal Privacy and Public Health, Mayo Clin. Proc. (2020). doi:10.1016/j.mayocp.2020.04.031.

[39] S. Cannicott, The ethics of contact tracing apps: International perspectives, Cent. Data Ethics Innov. Blog. (2020). https://cdei.blog.gov.uk/2020/05/12/the-ethics-of-contact-tracing-apps-internationalperspectives/ (accessed June 12, 2020).

[40] P. Sapiezynsk, J. Pruessing, V. Sekara, The Fallibility of Contact-Tracing Apps, (2020). https://arxiv.org/abs/2005.11297v3.

[41] O. Park, Y.J. Park, S.Y. Park, Y.M. Kim, J. Kim, J. Lee, E. Park, D. Kim, B.H. Jeon, B. Ryu, D. Ko, E. Kim, H. Kim, H. Lee, J. Gwack, J. Jo, J.H. Lee, J. Hyun, J. Kim, J.K. Park, S. Lee, S.S. Kim, S.H. Shin, S.W. Choi, T. Kim, U.N. Kim, Y. Woo, Y. Jin, Y.S. Jang, Y. Park, M. Yum, Contact transmission of Covid-19 in South Korea: Novel investigation techniques for tracing contacts, Osong Public Heal. Res. Perspect. (2020). doi:10.24171/j.phrp.2020.11.1.09.

[42] L. Franceschi-Bicchierai, The 10 Biggest Revelations From Edward Snowden's Leaks, Mashable.Com. (2014). https://mashable.com/2014/06/05/edward-snowden-revelations/?europe=true (accessed June 29, 2020).

[43] H. Cho, D. Ippolito, Y.W. Yu, Contact Tracing Mobile Apps for COVID-19: Privacy Considerations and Related Trade-offs, ArXiv:2003.11511. (2020). http://arxiv.org/abs/2003.11511.

[44] Jumbo Privacy, Jumbo Privacy Review: North Dakota’s Contact Tracing App, (2020). https://blog.jumboprivacy.com/jumbo-privacy-review-north-dakota-s-contact-tracing-app.html (accessed June 12, 2020).

[45] Amnesty International, Bahrain, Kuwait and Norway contact tracing apps among most dangerous for privacy, Amnesty.Org. (2020). https://www.amnesty.org/en/latest/news/2020/06/bahrain-kuwaitnorway-contact-tracing-apps-danger-for-privacy/ (accessed June 17, 2020).

[46] K. Bock, C.R. Kühne, R. Mühlhoff, M.R. Ost, J. Pohle, R. Rehak, Data Protection Impact Assessment for the Corona App, SSRN Electron. J. (2020). doi:10.2139/ssrn.3588172.

[47] G. Gopinath, The Great Lockdown: Worst Economic Downturn Since the Great Depression, Int. Monet. Fund. (2020). https://blogs.imf.org/2020/04/14/the-great-lockdown-worst-economic-downturn-sincethe-great-depression/ (accessed June 12, 2020).

[48] OECD, OECD Economic Outlook, Interim Report March 2020, 2020. doi:10.1787/7969896b-en.

[49] M. Klenk, H. Duijf, C. Engels, Ethics of Digital Contact Tracing and COVID-19: Who Is (Not) Free to Go?, SSRN Electron. J. (2020). doi:10.2139/ssrn.3595394.

[50] E. Woollacott, Coronavirus Tracing App Shared Data With Game Show, Forbes. (2020). https://www.forbes.com/sites/emmawoollacott/2020/06/16/coronavirus-tracing-app-shared-data-withgame-show/\#3dde4dab68e2 (accessed June 17, 2020).

[51] Amesty International UK, Qatar: "huge" security weakness in COVID-19 contact-tracing app, (2020). https://www.amnesty.org.uk/press-releases/qatar-huge-security-weakness-covid-19-contact-tracingapp?utm_source=google\&utm_medium=grant\&utm_campaign=AWA_GEN_coronavirus-dynamicsearch-ads\&utm_content $=($ accessed June 12,2020$)$.

[52] A.B. Dar, A.H. Lone, S. Zahoor, A.A. Khan, R. Naaz, Applicability of Mobile Contact Tracing in Fighting Pandemic (COVID-19): Issues, Challenges and Solutions, J. Cryptol. (2020). https://eprint.iacr.org/2020/484.

[53] D. Antonioli, N.O. Tippenhauer, K. Rasmussen, BIAS: Bluetooth Impersonation AttackS, Proc. IEEE Symp. Secur. Priv. (2020). doi:10.1109/SP40000.2020.00093.

[54] A. Laughlin, More than one billion Android devices at risk of malware threats, Which.Co.Uk. (2020). 
https://www.which.co.uk/news/2020/03/more-than-one-billion-android-devices-at-risk-of-malwarethreats/ (accessed June 14, 2020).

[55] A. Holst, Number of smartphone users worldwide from 2016 to 2021 (in billions), Statista.Com. (2019).

[56] Statista, Share of global smartphone shipments by operating system from 2014 to 2023, Statista.Com. (2020). https://www.statista.com/statistics/272307/market-share-forecast-for-smartphone-operatingsystems/ (accessed April 18, 2020).

[57] D. Luu, How out of date are Android devices?, Danluu.Com. (2020). https://danluu.com/androidupdates/ (accessed April 25, 2020).

[58] GSMA, GSMA Mobile Economy 2020, 2020. https://www.gsma.com/mobileeconomy/wpcontent/uploads/2020/03/GSMA_MobileEconomy2020_Global.pdf.

[59] Our World in Data, Mobile phone subscriptions vs. GDP per capita, 2017, Ourworldindata.Org. (2017). https://ourworldindata.org/grapher/mobile-phone-subscriptions-vs-gdp-per-capita (accessed June 29, 2020).

[60] Office for National Statistics, Internet access - households and individuals, Great Britain: 2019, 2019. https://www.ons.gov.uk/peoplepopulationandcommunity/householdcharacteristics/homeinternetandsoci almediausage/bulletins/internetaccesshouseholdsandindividuals/2019\#main-points.

[61] Statista, Smartphone ownership in the United Kingdom (UK) 2012-2020, by age, Statista. (2020). https://www.statista.com/statistics/271851/smartphone-owners-in-the-united-kingdom-uk-by-age/.

[62] Georgios Magklaras, L. Nikolaia, L. Bojorquez, A review of information security aspects of the emerging COVID-19 contact tracing mobile phone applications, ArXiv.Org. (2020). https://arxiv.org/abs/2006.00529v1.

[63] S. Biddle, The Inventors of Bluetooth Say There Could Be Problems Using Their Tech for Coronavirus Contact Tracing, Intercept. (2020). https://theintercept.com/2020/05/05/coronavirus-bluetooth-contacttracing/ (accessed June 16, 2020).

[64] OFCOM, Adults' media use and attitudes report 2020, (2020). https://www.ofcom.org.uk/research-anddata/media-literacy-research/adults/adults-media-use-and-attitudes (accessed June 30, 2020).

[65] P.A.M. Van Lange, J. Joireman, C.D. Parks, E. Van Dijk, The psychology of social dilemmas: A review, Organ. Behav. Hum. Decis. Process. 120 (2013) 125-141. doi:10.1016/j.obhdp.2012.11.003.

[66] C. Thöni, S. Volk, Conditional cooperation: Review and refinement, Econ. Lett. 171 (2018) 37-40. doi:10.1016/j.econlet.2018.06.022.

[67] S. Trang, M. Trenz, W.H. Weiger, M. Tarafdar, C.M.K. Cheung, One app to trace them all? Examining app specifications for mass acceptance of contact-tracing apps, Eur. J. Inf. Syst. (2020) 1-14. doi:10.1080/0960085X.2020.1784046.

[68] E.T. Straub, Understanding Technology Adoption: Theory and Future Directions for Informal Learning, Rev. Educ. Res. 79 (2009) 625-649. doi:10.3102/0034654308325896.

[69] R.A. Calvo, S. Deterding, R.M. Ryan, Health surveillance during covid-19 pandemic, BMJ. (2020) m1373. doi:10.1136/bmj.m1373.

[70] S.N. Williams, C.J. Armitage, T. Tampe, K. Dienes, Public attitudes towards COVID-19 contact tracing apps: A UK-based focus group study, MedRxiv. (2020). doi:https://doi.org/10.1101/2020.05.14.20102269.

[71] Emma Bradshaw, R. Ryan, M. Noetel, A. Saeri, P. Slattery, E. Grundy, R. Calvo, Information safety assurances affect intentions to use COVID-19 contact tracing applications, regardless of autonomysupportive or controlling message framing, (2020). doi:https://doi.org/10.31219/osf.io/5wap8.

[72] P. Sheeran, T.L. Webb, The Intention-Behavior Gap, Soc. Personal. Psychol. Compass. 10 (2016) 503518. doi:10.1111/spc3.12265.

[73] D. Kaafar, V. Teague, Y. Yarom, Joint Statement on Contact Tracing: Date 19th April 2020, (2020) 19. https://drive.google.com/file/d/1OQg2dxPu-X-RZzETlpV31Fa259Nrpk1J/view (accessed April 24, 
2020).

[74] Y. Bengio, R. Janda, Y.W. Yu, D. Ippolito, M. Jarvie, D. Pilat, B. Struck, S. Krastev, A. Sharma, The need for privacy with public digital contact tracing during the COVID-19 pandemic, Lancet Digit. Heal. (2020). doi:10.1016/S2589-7500(20)30133-3.

[75] F. Shi, J. Wang, J. Shi, Z. Wu, Q. Wang, Z. Tang, K. He, Y. Shi, D. Shen, Review of Artificial Intelligence Techniques in Imaging Data Acquisition, Segmentation and Diagnosis for COVID-19, IEEE Rev. Biomed. Eng. (2020) 1-1. doi:10.1109/RBME.2020.2987975.

[76] A.S.R. Srinivasa Rao, J.A. Vazquez, Identification of COVID-19 can be quicker through artificial intelligence framework using a mobile phone-based survey when cities and towns are under quarantine, Infect. Control Hosp. Epidemiol. 41 (2020) 826-830. doi:10.1017/ice.2020.61.

[77] D.S.W. Ting, L. Carin, V. Dzau, T.Y. Wong, Digital technology and COVID-19, Nat. Med. 26 (2020) 459-461. doi:10.1038/s41591-020-0824-5.

[78] T. Yang, M. Gentile, C.F. Shen, C.M. Cheng, Combining point-of-care diagnostics and internet of medical things (IOMT) to combat the Covid-19 pandemic, Diagnostics. (2020). doi:10.3390/diagnostics10040224.

\section{Declarations}

\section{Funding}

This research did not receive any specific grant from funding agencies in the public, commercial, or not-forprofit sectors.

\section{Conflict of Interest}

None declared.

\section{Availability of data and material}

Not applicable.

\section{Code availability}

Not applicable.

\section{Ethics approval}

Not applicable.

\section{Consent to participate}

Not applicable. 